\title{
New Insights Involving the Home Team Advantage
}

\author{
Tim B. Swartz and Adriano Arce*
}

\begin{abstract}
Although the home team advantage is known to exist in many sports, there are nuances of the advantage that are less well understood. In this paper, we investigate various aspects of the home team advantage including changes in the advantage over time, the relationship of the advantage to the overall scoring rate and differential advantages within leagues. The analysis is mainly based on descriptive statistics and is confined to the home team advantage pertaining to the National Hockey League and the National Basketball Association.
\end{abstract}

Keywords: National Basketball Association, National Hockey League, sporting myths.

*Tim Swartz is Professor in the Department of Statistics and Actuarial Science, Simon Fraser University, 8888 University Drive, Burnaby BC, Canada V5A1S6. Swartz has been partially supported by grants from the Natural Sciences and Engineering Research Council of Canada (NSERC). Adriano Arce is an undergraduate student at Simon Fraser University who has been partially supported by NSERC's Undergraduate Student Research Awards (USRA) program. The authors thank two reviewers whose comments led to a significant improvement in the manuscript. 


\section{INTRODUCTION}

The home team advantage is a phenomenon whereby sports teams experience a competitive benefit from playing at their home venue. Whereas the home team advantage has been known to exist in sport for a very long time, the underlying causes are the subject of much discussion (Entine and Small 2008). For years, people have speculated that the home team advantage is a result of a number of factors including travel fatigue with respect to the visiting team, home team spectator support and home team familiarity (Stefani 2008). Nevill, Balmer and Wolfson (2005) provide an overview of the traditional views regarding home team advantage. More recently, a number of investigators have argued that the home team advantage is primarily due

to officiating bias. A summary of these research findings are provided in chapters 10 and 11 of the entertaining book by Moskowitz and Wertheim (2011). Some evidence of biased officiating in the National Hockey League (NHL) is given in Remark \#2 of Beaudoin and Swartz (2010).

The extent of the home team advantage varies from sport to sport. For example, the home ice advantage in the NHL yields a winning percentage of $54.5 \%$ during the regular season compared to the home court advantage of $60.5 \%$ in the National Basketball Association (NBA); see Swartz et al. (2011). Because the home team advantage is so beneficial, many leagues structure an incentive during the regular season. The incentive is that an extra home game is assigned in playoff series to teams with the better regular season record should the series extend to the maximum number of matches.

In this paper, we look at a number of subtle issues related to the home team advantage. We restrict our analysis to the home team advantage as it pertains to the NHL and the NBA, two of the four prominent professional leagues in North America. The games of hockey (NHL) and basketball (NBA) share similarities in that they are continuous free flowing contests where referees constantly assess whether penalties/fouls should be assigned. This is in contrast to the two other major North American sports of football (National Football League) and baseball 
(Major League Baseball) which may be viewed as discrete games where short bursts of action are followed by longer periods of inaction. One of the novelties of our approach is that we consider scoring differentials in our analyses rather than traditional win/loss data when assessing home team advantage. It is a generally accepted statistical principle that data are valuable and that one should not "waste" data by needless summarization. Our analysis is primarily confined to descriptive statistics.

In section 2 , we begin by investigating the change in the magnitude of the home team advantage in the NHL and the NBA over the last 33 seasons. We observe that there is a general decrease in the extent of the home team advantage. However, when adjusted for scoring output, the decline in home advantage is barely detectable. Doyle and Leard (2012) considered a smaller window for the NHL (six seasons from 2005/06 through 2010/11) and introduced various covariates for the assessment of home ice advantage. They found no clear trend in the variation of home ice advantage over time. If we adopt the view that the home team advantage is primarily due to officiating bias, then a reduction in the magnitude of the home team advantage is consistent with the increasing scrutiny that NHL and NBA officials have received in recent years (Price and Wolfers 2010). With the advent of modern technology for review purposes, officiating review panels, etc, it is conceivable that there has been a reduction in officiating bias.

In section 3, we revisit the question of differential home team advantages. That is, we investigate whether some teams in a league have persistently higher/lower advantages than other teams. The complicating aspect of the analysis is that it involves a multiple comparisons issue. Even if all teams in a league have the same home team advantage, natural variation will cause some teams to appear to have higher/lower advantages than other teams. A key aspect of our analysis is that we remove the effect of the annual league scoring rate from the home team advantage. In addition, we highlight the importance of elevation with respect to the home team advantage. Excluding effects due to elevation, we essentially conclude that there are no 
differential home team advantages. In the previously mentioned NHL study, Doyle and Leard (2012) also concluded that the home ice advantage does not appear to differ significantly across teams. For basketball, in a study of the 1991/92 college season (NCAA), Harville and Smith (1994) found that the team-to-team differences in home court advantage were relatively small. For the NBA, Jones (2008) studied four seasons (2002/03 through 2005/06) with an emphasis on the home court advantage in each quarter of play. He provided a nuanced conclusion regarding team specific differences based on the reliability of difference scores involving positively correlated variables. In English soccer, Clarke and Norman (1995) found only mild evidence for the existence of differential home field advantages. We end with a short discussion in section 4.

We now discuss some of the related work of Richard Pollard who has made significant contributions to the investigation of the home team advantage. Much of Pollard's work attempts to identify factors that are contributory to the home advantage. Pollard's approach is generally characterized by regressing a home team advantage response variable against covariates of interest. In Pollard (2002), the effect of moving to a new stadium within the same city was seen to reduce the home advantage through the study of 37 major North American sports teams. Change of venue may be viewed as an aspect of "familiarity" which has been attributed as a factor in the home team advantage. In an investigation of world soccer, Pollard (2006) concluded that territory plays a role in the home team advantage where some broad geographical areas exhibited greater home field advantages than other broad geographical areas. In Gomez and Pollard (2011), European basketball was studied where it was found that there was variation in the home court advantage amongst countries and that capital cities had a lesser home advantage. Corroborating our findings in section 2, Pollard and Gomez (2009) observed a decrease in home field advantage in domestic European soccer (France, Italy, Portugal and Spain) based on studies beginning as early as the 1928-1929 season up until 2006-2007. The decreasing trend is most prominent from roughly 1980 onwards. The authors provide a number of plausible explanations for the decline. In contrast to our findings in section 3, Pollard and 
Gomez (2009) observed differential home advantages for soccer teams in France, Italy and Portugal. The authors point to historical, ethnic, cultural and territorial influences, factors that are less prominent in the North American sports considered in this paper. Most directly related to our work, Pollard and Pollard (2005) also studied the home advantage in the NHL and the NBA over time. They concluded that there has been a decline in the home advantage from about 1980 onwards. One of the differences between their work and ours is that our study provides an update for the ten seasons 2002-2003 through 2011-2012. In addition, we provide an alternative definition of home team advantage that analyzes scoring differentials rather than binary win/loss data. We also take into account league scoring totals which are strongly correlated with the home team advantage. This is something that does not seem to have been previously considered in the literature concerning the home team advantage.

\section{THE HOME TEAM ADVANTAGE OVER TIME}

For both the NHL and the NBA, data were obtained from the website www.shrpsports.com. To process the data into a manageable form, script writing was carried out using a combination of R, Python, Java and iMacros.

Although data were available even further back in time, we collected NHL and NBA regular season data from 1979/80 through 2011/12. In the NHL, 1979/80 was the first season following the merger of the NHL with the World Hockey Association (WHA). We note that NHL data were unavailable in 2004/05 due to the labour lockout which resulted in a cancelled season. In the NBA, 1979/80 was the inaugural season of the three-point shot.

For both leagues we obtained a season specific home team advantage variable HTA which was calculated by averaging the total home goals (points) less the total away goals (points) over all games for each season. The interpretation of HTA is that it is the number of goals (NHL) and points $(\mathrm{NBA})$ by which an average home team defeats an average road team. Note that 
NHL and NBA schedules are not completely balanced; i.e. a team may not play every opponent an equal number of times in home and away matches. However, we expect this lack of balance to have a small effect in measuring home team advantage.

In Figure 1, we plot the home team advantage variable HTA versus season. We observe a stunning pattern in both leagues where home team advantage has decreased over time. In the NHL, if we take the 32 values and carry out a one-sided $t$-test, the year effect is strongly significant with a $p$-value of $2.71 * 10^{-14}$. In the NBA, the $p$-value is $1.10 * 10^{-16}$. The decline in home advantage is very important to the sports gambling industry where pointspreads are assigned to every game. It would be clearly wrong to assume that the home team advantage has been constant over time. Looking at Figure 1 more closely, it appears that in both leagues, the downward trend was greater from 1980-1994 than from 1995-2012.
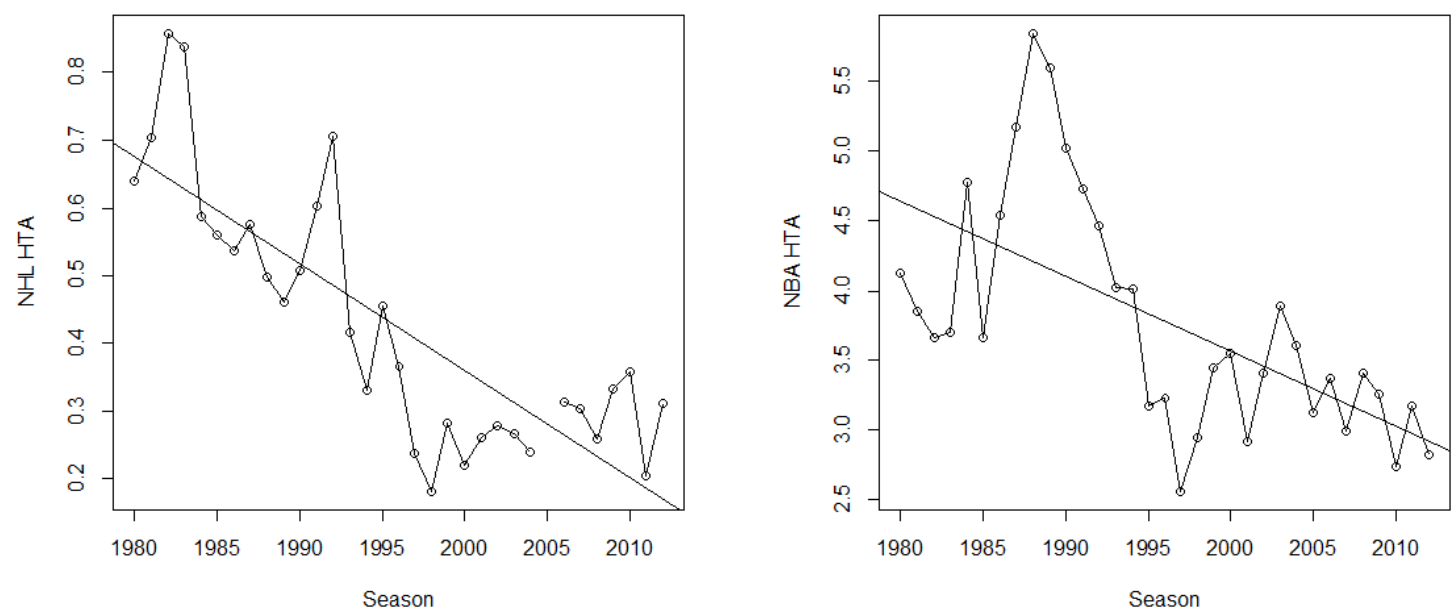

Figure 1: Plots of the NHL home ice advantage versus season and the NBA home court advantage versus season based on scoring differentials. Linear fits are included to help assess the overall trend.

Admittedly, there are alternative measures of the home team advantage. For example, 
it is clear that a team's objective is to win matches rather than maximize score differential. Therefore in the NBA, we also consider the seasonal winning proportion $H / N$ as an appropriate measure of home advantage where $H$ is the number of wins by home teams and $N$ is the number of matches in a season. Using this measure, the value 0.50 confers no home advantage. For the NHL, we consider $(2 H+T) /(2 N)$ where $H$ is the number of home wins in regulation time and $T$ is the number of ties in regulation time. Also, as pointed out by a Reviewer, when relating home advantage to underlying factors, scoring differential may not be linearly related to various factors of interest. For example, Garicano, Palacios-Huerta and Prendergast (2005) found that the referee's decision to add minutes to the end of soccer matches depended on the score of the match. Referees tended to limit added minutes when the score differential was slightly in favor of the home team. In Figure 2, we provide analogous plots to Figure 1 using these alternative measures of home advantage. Qualitatively, Figure 1 and Figure 2 have similar characteristics.
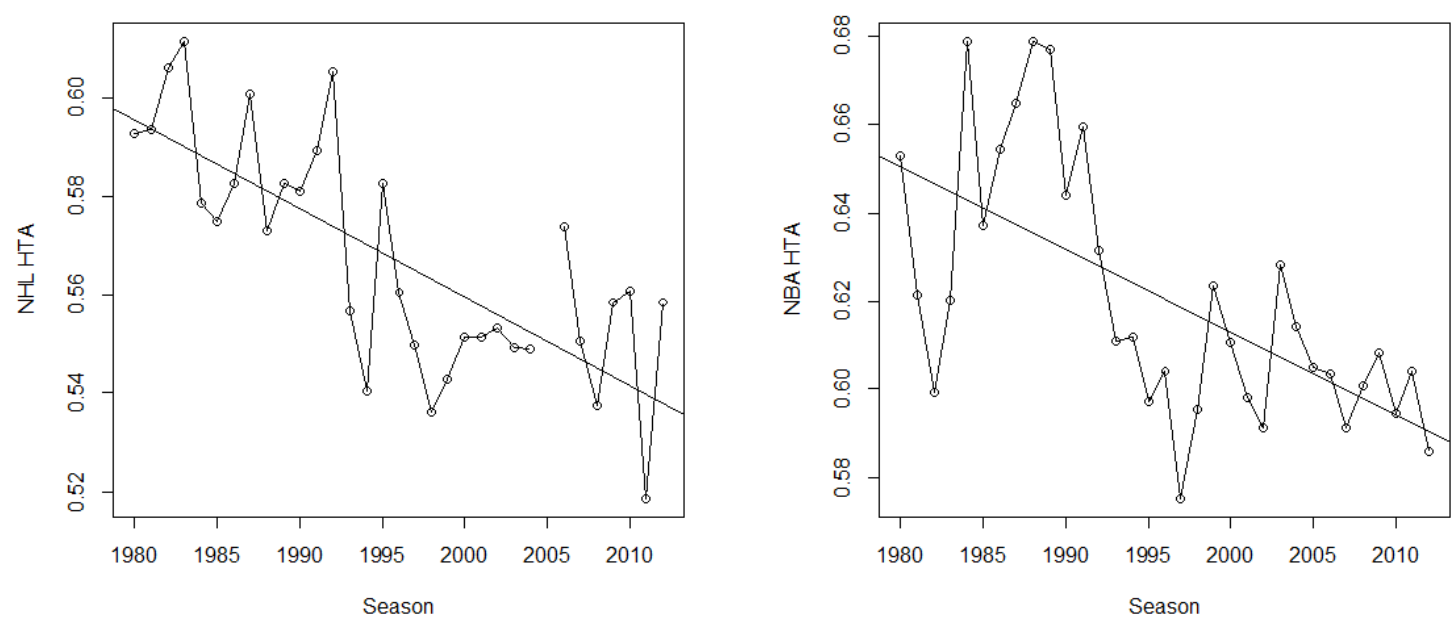

Figure 2: Plots of the NHL home ice advantage versus season and the NBA home court advantage versus season based on winning proportions. Linear fits are included to help assess the overall trend. 
To help explain Figure 1, we obtained an additional season specific variable TPT (total points per game) which was calculated by averaging the total points (goals) scored by all teams over all games for each season. In Figure 3, we plot TPT versus season and similarly observe a general decreasing pattern over time. It is understandable that TPT may have seasonal variation. For example, there were radical rule changes in the NHL following the 2004/05 lockout which encouraged offense. Also, leagues go through cycles where defense/offense is emphasized such as 1998/99 through 2003/04 in the NBA. This may have been an attempt to emulate the highly successful teams such as the San Antonio Spurs who specialized in defense. In a comparison of Figure 1 and Figure 3, the 2012 home advantage in the NHL accounts for roughly $5 \%$ of goals ( 0.3 goals out of 5.5 total goals scored). In the NBA, the 2012 calculation is roughly $2 \%$ (3.5 points out of 190 total points).
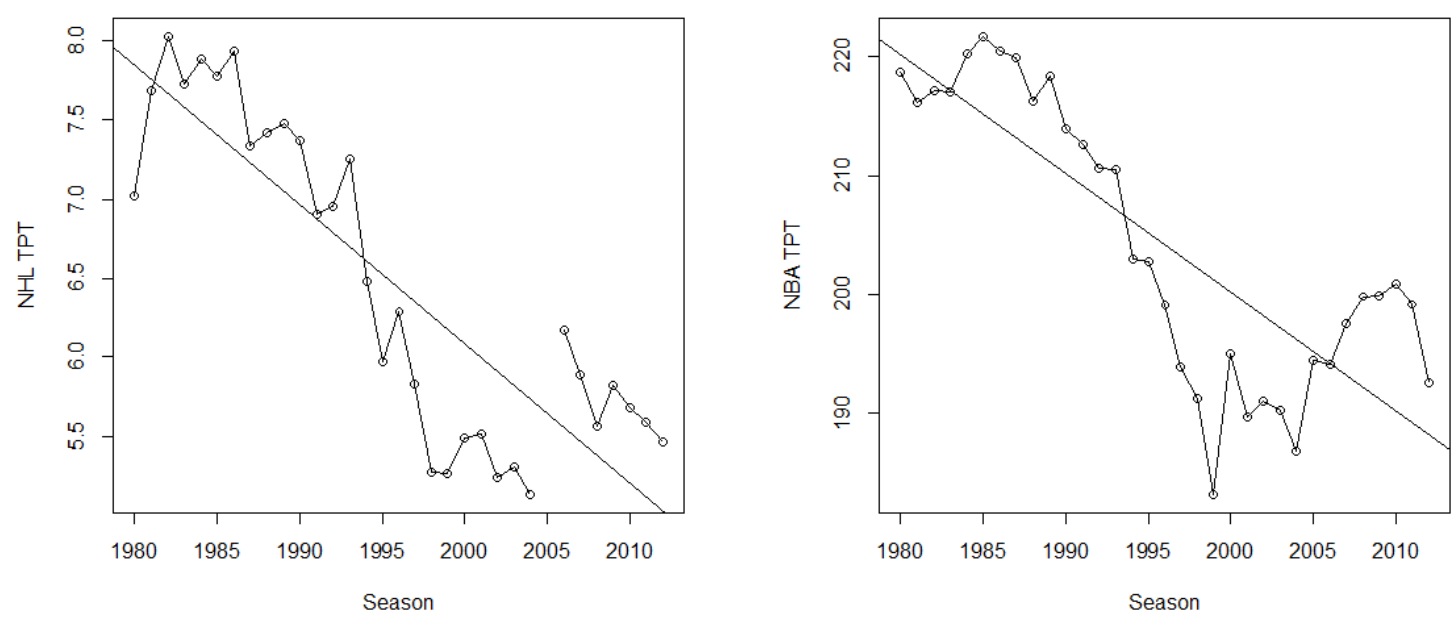

Figure 3: Plots of the NHL goals per game versus season and the NBA points per game versus season. Linear fits are included to help assess the overall trend.

Returning to the comparison between Figure 1 and Figure 3, it appears that home team advantage (HTA) and total points (TPT) are correlated. This is borne out by the sample 
correlation coefficients $r=0.86$ for the NHL and $r=0.70$ for the NBA. In retrospect, the positive correlation may not be surprising as one might expect home team advantage to be magnified in high scoring seasons. For example, in seasons where teams play at a higher tempo (i.e. more possessions), the scoring advantage of the stronger team ought to be proportional to the number of possessions.

To further investigate the effect of home team advantage, we regress HTA against TPT for both leagues. The residuals represent the unexplained variation in HTA after TPT has been removed, and we plot the residuals versus season in Figure 4. For the NHL, the linear fit gives a slope estimate of -0.0014 with standard error 0.0018. For the NBA, the linear fit gives a slope estimate of -0.0049 with standard error 0.0111. The trends are not statistically significant, and this suggests that after accounting for total scoring, there has not been a decline in home advantage over the last 33 years. Alternatively, if we believe that the negative estimated slopes suggest a mild decline in home advantage, we ask how this might be explained? If we accept the Moskowitz and Wertheim (2011) hypothesis that home team advantage is largely due to officiating bias, then it is plausible that officiating bias has decreased slightly over time in the modern era. A rationale for this is that referees have been subject to increasing scrutiny with video replays, officiating review panels, and so on. It may be the case that officiating is improving, and part of this may be self-preservation on the part of officials.

Of course, the factors influencing home advantage are numerous, complex and difficult to disentangle. Therefore, the decline in the home team advantage as observed in the NHL and the NBA can be logically attributed to a reduction in officiating bias only if we believe that officiating bias is the sole underlying cause (Moskowitz and Wertheim, 2011). Various underlying factors (territoriality, culture, history and playing conditions) that have been associated with the home advantage are most likely less relevant in North American sporting leagues such as the NHL and the NBA where conditions are more homogeneous. 

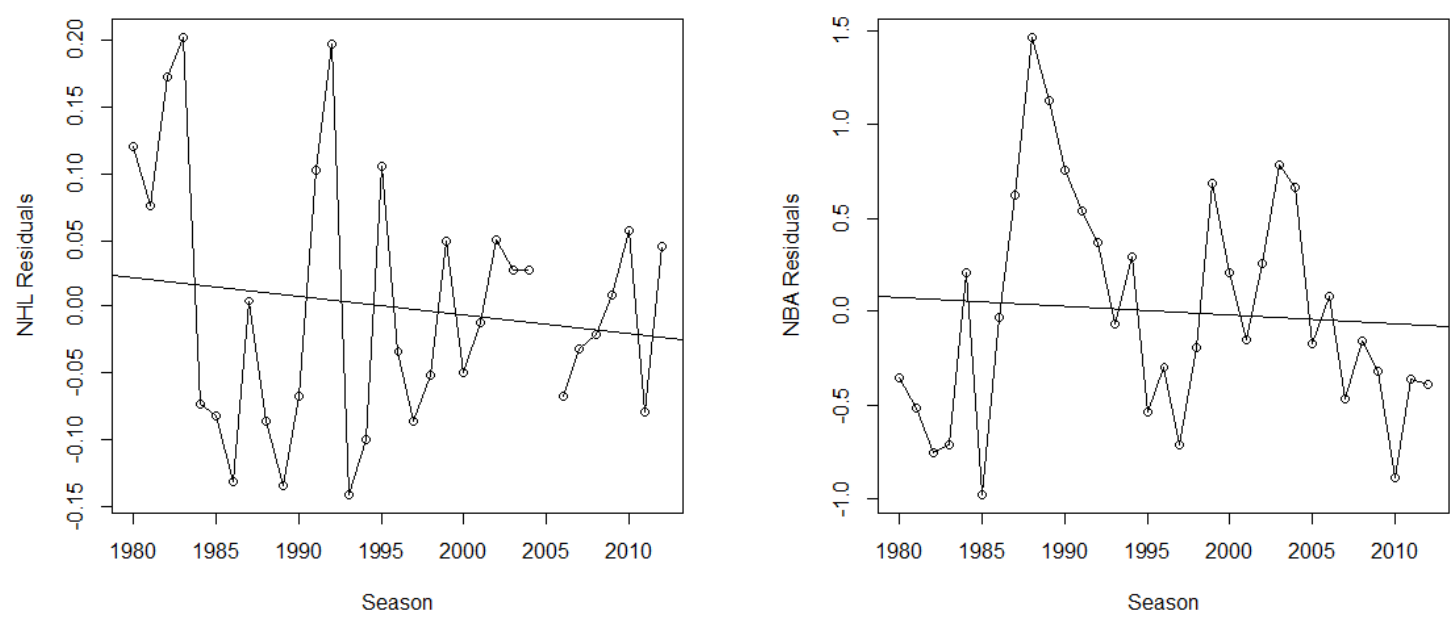

Figure 4: Residual plots for the NHL and NBA after regressing HTA versus TPT. Linear fits are included to help assess the overall trend.

\section{DIFFERENTIAL HOME TEAM ADVANTAGES}

The question that we investigate in this section is whether the magnitude of the home team advantage varies across teams in a league. The common perception is that there are teams for whom a significantly larger advantage exists. A quick internet search provides no shortage of quotes of the following type:

- As they have many times before, the Storm were voted to have the best home-court advantage in the WNBA (Women's National Basketball Association) with 82 percent of the vote. (www.heraldnet.com/article/20120517/BLOG51/120519786)

Before proceeding to the analysis, there are a few comments worthy of consideration. First, if a team truly has an extraordinary home team advantage, it would seem reasonable that the advantage should exist beyond a single season. Second, given a sports league, natural variation in data will always result in observed differential team advantages even when there is no real 
effect. This leads to the statistical problem of multiple comparisons. Third, it is a well-known psychological phenomenon that people tend to remember exceptional events (Schmidt 2012). For example, if a team goes undefeated at home in a season, they will be remembered by many as having an incredible home team advantage.

For our analysis, we use the same NHL and NBA datasets as described in section 2. When a franchise moves to a different city, we impose a demarcation which defines two distinct teams. The logic is that the traditional reasons for home advantage (travel issues, spectator support and home familiarity) are subject to change when a franchise moves to a different city. When a team changes its name or its venue within a city, we treat the team as a single entity. With these definitions, our datasets give rise to 37 NHL teams and 36 NBA teams.

Consider the $i$ th NHL team in its $j$ th season. We can define its home team advantage $\mathrm{HTA}_{i j}$ as its average goal differential in home games less its average goal differential in road games. Note that this is strictly a measure of home ice advantage and does not depend on the quality of the team. For example, a very bad team could be outscored by 1.5 goals on average at home and be outscored by 2.0 goals on average on the road during a season. However, this team would still have a positive home ice advantage of $-1.5-(-2.0)=0.5$ goals. To account for seasonal scoring rates (see section 2), we define $\mathrm{TPT}_{j}$ as the total goals per game in season $j$. $\mathrm{TPT}_{j}$ is calculated by averaging the total goals scored by all teams in the league over all games in the $j$ th season. Accordingly, we define the standardized home team advantage for the $i$ th team in the $j$ th season as

$$
\mathrm{SHTA}_{i j}=\frac{\mathrm{HTA}_{i j}}{\mathrm{TPT}_{j}}
$$

The statistic $\mathrm{SHTA}_{i j}$ is the quantity of interest in assessing differential home ice advantage in the NHL. An analogous statistic is calculated for the NBA data. In Table 1 (NHL) and Table 2 (NBA), we provide summary information on all of the teams. For the 16 teams that 
have participated in at least 30 seasons in the NHL, we observe that Pittsburgh and Calgary have the highest average SHTA. For the 20 teams that have participated in at least 30 seasons in the NBA, we observe that Denver and Utah have the highest average SHTA.

To get a sense of the variability in our data, we first consider teams in Table 1 and Table 2 who have participated in at least 30 seasons. From the NHL teams, we identify Boston and Pittsburgh who have the smallest and largest SHTA averaged over all seasons. In the NBA, we similarly identify Philadelphia and Denver. In Figure 5, we plot the seasonal SHTA statistic for these teams together with the league average SHTA. Our visual impression is that perhaps only Denver has a markedly different home team advantage when compared to the NBA league average.

As in section 2, we are interested in seeing if similar results are obtained under an alternative definition of home advantage. We therefore averaged the yearly home winning proportion for each team over all seasons. We then calculated the correlation coefficient between this variable and SHTA over each of the 16 NHL teams that have participated in at least 30 seasons. We did likewise for the 20 NBA teams that have participated in at least 30 seasons. We obtained $r=0.917$ for the NHL and $r=0.889$ for the NBA which indicates that there is strong agreement between the two measures of home advantage.

There is another curiousity in our analysis. If we consider the NHL and NBA cities in our dataset, it turns out that all of the cities except three have elevations ranging from sea level to 2200 feet. The three exceptions are Denver (5130 feet), Salt Lake (4330 feet) and Calgary (3440 feet). Corresponding to Table 1, we note that the Colorado Rockies, the Colorado Avalanche and the Calgary Flames are ranked 2nd, 32nd and 5th respectively amongst the 37 NHL teams in terms of average SHTA. Corresponding to Table 2, we note that the Denver Nuggets and the Utah Jazz are ranked 1st and 2nd respectively amongst the 36 NBA teams in terms of average SHTA. It therefore seems that elevation provides an edge in the home team advantage, especially in the NBA. Although the edge has been discussed for years with respect to the 

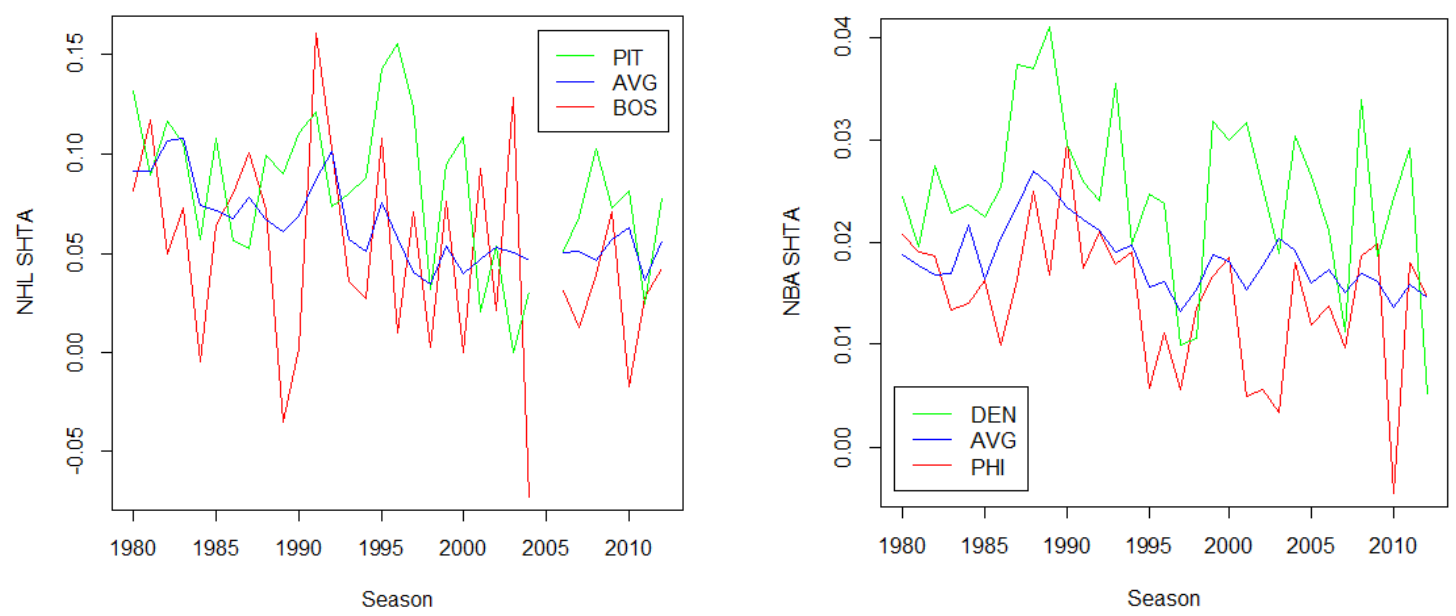

Figure 5: Plots of the weakest home team advantage (red line), average home team advantage (blue line) and strongest home team advantage (green line) for both the NHL and NBA. In the NHL, the weakest/strongest team is Boston/Pittsburgh, and in the NBA, the weakest/strongest team is Philadelphia/Denver.

"mile high city" Denver, the advantage does not appear to be as widely appreciated for the other two cities (Salt Lake City, Calgary). An explanation for the observation is that the partial pressure of oxygen is reduced at higher altitudes, and therefore physical adaptations are required. Consequently, teams that live, train and compete in Denver (for example) have adapted whereas visiting teams to Denver may not have adapted. High altitude training has been a long-time technique in many endurance sports. In South American soccer, Pollard (2005) notes that Bolivia has the largest domestic home field advantage where its largest city La Paz resides at 11,500 feet elevation.

It has been pointed out that the elevation variable may be confounded with travel fatigue as Denver, Utah and Calgary are travel locations that will often involve a change of time zone for the visiting team. The impact of travel on the home team advantage is interesting and complex. In addition to crossing time zones, cumulative travel may be important and this takes on issues 
such as clearing customs, the availability of direct flights, distinguishing teams that charter their own planes, league scheduling, etc. We do note that some NBA locations notorious for troublesome travel (e.g. Minnesota, Vancouver, Oklahoma City, San Antonio, Kansas City) do not have teams with sizeable home court advantages.

Inferentially, we can view the SHTA data in a one-way anova context where the teams are treatments and there are an unequal numbers of replications across treatments. Restricting the analysis to the 16 NHL teams and the 20 NBA teams who have completed at least 30 seasons, we first tested for the equality of teams. The NHL data gave the statistic $F_{15,495}=1.61$ with a $p$-value of 0.067 . The NBA data gave the statistic $F_{19,639}=5.47$ with a highly significant $p$-value of $1.48 * 10^{-12}$. We then made pairwise comparisons using Tukey's HSD method. For the NHL, all teams clustered together at level 0.05 significance. That is, there is no evidence of pairwise differences amongst any of the 16 NHL teams. For the NBA, Tukey's HSD method produced two clustering labels. In terms of SHTA, all of the 20 teams except Denver and Utah received the lower clustering label. Out of interest, we repeated the analysis for the more recent seasons 1994-1995 through 2011-2012. We again observed two clustering labels where all of the 20 teams except Denver and Utah received the lower clustering label.

\section{DISCUSSION}

We have provided an exploration of the home team advantage as it exists in the National Hockey League and the National Basketball Association. Viewing officiating bias as a primary factor in the home team advantage, these two leagues offer a good opportunity to detect small changes in the home team advantage since referees are under non-stop pressure to assess penalties in these continuous free flowing games. Our analyses are comprehensive in the sense that our datasets are large, and extend back to the 1979/80 seasons. We also consider more informative data than typical win percentage statistics; instead we take goal (NHL) and point (NBA) differentials into account. 
In both leagues, we have observed a general decrease in the home team advantage over time, and this has coincided with a general decrease in the league scoring rates. When the correlation is taken into account, there appears at most to be a subtle decrease in the home team advantage over time. We argue that this may provide an indication of a reduction in officiating bias over time. As a future project, it would be interesting to investigate the change in officiating outcomes in the NHL and the NBA as a function of time.

With respect to differential home team advantages, there appears to be little evidence of the widely held belief that some teams in a league have extraordinary home team advantages. An exception to this rule may involve teams whose home games are played at high elevations where a systematic benefit is conferred. Although this observation appears to be generally appreciated for the "mile high city" Denver, the finding is less well-known for major cities such as Salt Lake City and Calgary.

\section{REFERENCES}

Beaudoin, D. and Swartz, T.B. (2010). Strategies for pullie the goalie in hockey. The American Statistician, 64(3): 197-204.

Clarke, S.R. and Norman, J.M. (1995). Home ground advantage of individual clubs in English soccer. The Statistician, 44(4): 509-521.

Doyle, J.M. and Leard, B. (2012). Variations in home advantage: evidence from the National Hockey League. Journal of Quantitative Analysis in Sports, 8(2), Article 5.

Entine, O.A. and Small, D.S. (2008). The role of rest in the NBA home-court advantage. Journal of Quantitative Analysis in Sports, 4(2), Article 6.

Garicano, L., Palacios-Huerta, I. and Prendergast, C. (2005). Favoritism under social pressure. The Review of Economics and Statistics, 87(2), 208-216.

Gomez, M.A. and Pollard, R. (2011). Reduced home advantage for basketball in teams in capital cities in Europe. European Journal of Sport Science, 11(12): 143-148.

Harville, D.A. and Smith, M.H. (1994). The home-court advantage: how large is it, and does it vary from team to team? The American Statistician, 48(1): 22-28. 
Jones, M.B. (2008). A note on team-specific home advantage in the NBA. Journal of Quantitative Analysis in Sports, 4(3), Article 5.

Moskowitz, T.J. and Wertheim, L.J. (2011). Scorecasting: The Hidden Influences Behind how Sports are Played and Games are Won, Crown Archetype: New York.

Nevill, A., Balmer, N. and Wolfson, S. (2005). The extent and causes of home advantage: Some recent insights. Journal of Sports Sciences, 23(4): 335-336.

Pollard, R. (2002). Evidence of a reduced home advantage when a team moves to a new stadium. Journal of Sports Sciences, 20(12): 969-973.

Pollard, R. (2006). World wide variations in home advantage in association football. Journal of Sports Sciences, 24(3): 231-240.

Pollard, R. and Gomez, M.A. (2009). Home advantage in football in South-West Europe: Longterm trends, regional variation, and team differences. European Journal of Sport Science, 9(6): 341-352.

Pollard, R. and Pollard, G. (2005). Long term trends in home advantage in professional team sports in North America and England (1876-2003). Journal of Sports Sciences, 23(4): 337-350.

Price, J. and Wolfers, J. (2010). Racial discrimination among NBA referees. Quarterly Journal of Economics, 125(4): 1859-1887.

Schmidt, S.R. (2012). Extraordinary Memories for Exceptional Events, Psychology Press: London.

Stefani, R. (2008). Measurement and interpretation of home advantage. In Statistical Thinking in Sports, editors Ruud H. Koning and James H. Albert, Chapman \& Hall/CRC: Boca Raton, 203-216.

Swartz, T.B., Tennakoon, A., Nathoo, F., Tsao, M. and Sarohia, P.S. (2011). Ups and downs: team performance in best-of-seven playoff series. Journal of Quantitative Analysis in Sports, 7(4), Article 2. 


\begin{tabular}{l||r|c|c}
\multicolumn{1}{c|}{ Team } & $n$ & Seasons & Avg SHTA \\
\hline Winnipeg Jets 2 & 1 & $2011 / 12-2011 / 12$ & 0.132 \\
Colorado Rockies & 3 & $1979 / 80-1981 / 82$ & 0.097 \\
Minnesota North Stars & 14 & $1979 / 80-1992 / 93$ & 0.086 \\
Pittsburg Penguins & 32 & $1979 / 80-2011 / 12$ & 0.082 \\
Calgary Flames & 31 & $1980 / 81-2011 / 12$ & 0.081 \\
Winnipeg Jets 1 & 17 & $1979 / 80-1995 / 96$ & 0.078 \\
Quebec Nordiques & 16 & $1979 / 80-1994 / 95$ & 0.076 \\
Detroit Red Wings & 32 & $1979 / 80-2011 / 12$ & 0.074 \\
Buffalo Sabres & 32 & $1979 / 80-2011 / 12$ & 0.073 \\
Columbus Blue Jackets & 11 & $2000 / 01-2011 / 12$ & 0.073 \\
Hartford Whalers & 18 & $1979 / 80-1996 / 97$ & 0.071 \\
Minnesota Wild & 11 & $2000 / 01-2011 / 12$ & 0.068 \\
St. Louis Blues & 32 & $1979 / 80-2011 / 12$ & 0.067 \\
Los Angeles Kings & 32 & $1979 / 80-2011 / 12$ & 0.067 \\
Washington Capitals & 32 & $1979 / 80-2011 / 12$ & 0.066 \\
Montreal Canadiens & 32 & $1979 / 80-2011 / 12$ & 0.064 \\
Edmonton Oilers & 32 & $1979 / 80-2011 / 12$ & 0.064 \\
Nashville Predators & 13 & $1998 / 99-2011 / 12$ & 0.062 \\
Anaheim Ducks/Mighty Ducks & 18 & $1993 / 94-2011 / 12$ & 0.061 \\
Chicago Black Hawks/Blackhawks & 32 & $1979 / 80-2011 / 12$ & 0.061 \\
Philadelphia Flyers & 32 & $1979 / 80-2011 / 12$ & 0.060 \\
New York Islanders & 32 & $1979 / 80-2011 / 12$ & 0.059 \\
Tampa Bay Lightning & 19 & $1992 / 93-2011 / 12$ & 0.058 \\
New Jersey Devils & 29 & $1982 / 83-2011 / 12$ & 0.058 \\
Vancouver Canucks & 32 & $1979 / 80-2011 / 12$ & 0.056 \\
San Jose Sharks & 20 & $1991 / 92-2011 / 12$ & 0.055 \\
New York Rangers & 32 & $1979 / 80-2011 / 12$ & 0.054 \\
Dallas Stars & 18 & $1993 / 94-2011 / 12$ & 0.051 \\
Toronto Maple Leafs & 32 & $1979 / 80-2011 / 12$ & 0.051 \\
Boston Bruins & 32 & $1979 / 80-2011 / 12$ & 0.049 \\
Ottawa Senators & 19 & $1992 / 93-2011 / 12$ & 0.046 \\
Colorado Avalanche & 16 & $1995 / 96-2011 / 12$ & 0.041 \\
Phoenix Coyotes & 15 & $1996 / 97-2011 / 12$ & 0.038 \\
Atlanta Thrashers & 11 & $1999 / 00-2010 / 11$ & 0.037 \\
Carolina Hurricanes & 14 & $1997 / 98-2011 / 12$ & 0.036 \\
Florida Panthers & 18 & $1993 / 94-2011 / 12$ & 0.034 \\
Atlanta Flames & 1 & $1979 / 80-1979 / 80$ & 0.030 \\
& & & \\
Nand & &
\end{tabular}

Table 1: NHL summary including number of seasons $n$ and average standardized home team advantage over all seasons. Note that the 2004/05 season was cancelled due to a labour lockout. 


\begin{tabular}{l||r|c|c}
\multicolumn{1}{c|}{ Team } & \multicolumn{1}{c|}{ Seasons } & Avg SHTA \\
\hline Denver Nuggets & 33 & $1979 / 80-2011 / 12$ & 0.025 \\
Utah Jazz & 33 & $1979 / 80-2011 / 12$ & 0.025 \\
Sacramento Kings & 27 & $1985 / 86-2011 / 12$ & 0.023 \\
Golden State Warriors & 33 & $1979 / 80-2011 / 12$ & 0.021 \\
Portland Trail Blazers & 33 & $1979 / 80-2011 / 12$ & 0.021 \\
Cleveland Cavaliers & 33 & $1979 / 80-2011 / 12$ & 0.021 \\
Indiana Pacers & 33 & $1979 / 80-2011 / 12$ & 0.020 \\
Seattle Supersonics & 29 & $1979 / 80-2007 / 08$ & 0.020 \\
San Diego Clippers & 5 & $1979 / 80-1983 / 84$ & 0.020 \\
Phoenix Suns & 33 & $1979 / 80-2011 / 12$ & 0.020 \\
Orlando Magic & 23 & $1989 / 90-2011 / 12$ & 0.020 \\
San Antonio Spurs & 33 & $1979 / 80-2011 / 12$ & 0.019 \\
Kansas City Kings & 6 & $1979 / 80-1984 / 85$ & 0.019 \\
Washington Wizards/Bullets & 33 & $1979 / 80-2011 / 12$ & 0.018 \\
Milwaukee Bucks & 33 & $1979 / 80-2011 / 12$ & 0.018 \\
Atlanta Hawks & 33 & $1979 / 80-2011 / 12$ & 0.018 \\
Houston Rockets & 33 & $1979 / 80-2011 / 12$ & 0.018 \\
Charlotte Bobcats & 8 & $2004 / 05-2011 / 12$ & 0.018 \\
Los Angeles Clippers & 28 & $1984 / 85-2011 / 12$ & 0.017 \\
New Jersey Nets & 33 & $1979 / 80-2011 / 12$ & 0.017 \\
Memphis Grizzlies & 11 & $2001 / 02-2011 / 12$ & 0.017 \\
Los Angeles Lakers & 33 & $1979 / 80-2011 / 12$ & 0.017 \\
Chicago Bulls & 33 & $1979 / 80-2011 / 12$ & 0.016 \\
New Orleans/Oklahoma City Hornets & 2 & $2005 / 06-2006 / 07$ & 0.016 \\
Boston Celtics & 33 & $1979 / 80-2011 / 12$ & 0.016 \\
Miami Heat & 24 & $1988 / 89-2011 / 12$ & 0.016 \\
Dallas Mavericks & 32 & $1980 / 81-2011 / 12$ & 0.016 \\
New York Knicks & 33 & $1979 / 80-2011 / 12$ & 0.015 \\
Charlotte Hornets & 14 & $1988 / 89-2001 / 02$ & 0.015 \\
Detroit Pistons & 33 & $1979 / 80-2011 / 12$ & 0.015 \\
Philadelphia 76ers & 33 & $1979 / 80-2011 / 12$ & 0.015 \\
Minnesota Timberwolves & 23 & $1989 / 90-2011 / 12$ & 0.015 \\
Toronto Raptors & 17 & $1995 / 96-2011 / 12$ & 0.014 \\
New Orleans Hornets* & 8 & $2002 / 03-2011 / 12$ & 0.014 \\
Oklahoma City Thunder & 4 & $2008 / 09-2011 / 12$ & 0.013 \\
Vancouver Grizzlies & 6 & $1995 / 96-2000 / 01$ & 0.012 \\
& & & \\
Nand & &
\end{tabular}

Table 2: NBA summary including number of seasons $n$ and average standardized home team advantage over all seasons. *Note that for the 2005/06 to 2006/07 seasons, the New Orleans Hornets relocated to Oklahoma City due to Hurricane Katrina. 